\title{
An evaluation of coronary artery plaque burden in asymptomatic type 2 diabetics using dual- source CT coronary angiography
}

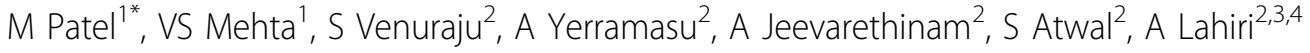 \\ From International Conference for Healthcare and Medical Students 2011 \\ Dublin, Ireland. 4-5 November 2011
}

\section{Introduction}

Current International Diabetes Federation (IDF) and American Diabetes Association (ADA) guidelines advocate investigation for coronary artery disease (CAD) in only those patients who have either typical/atypical cardiac symptoms or an abnormal resting ECG. As it is well established that diabetics with CAD may be asymptomatic, we hypothesised that a large proportion of asymptomatic diabetics may have coronary arterial plaques causing significant stenosis which goes undetected with current guidelines.

\section{Methods}

A cohort of 71 type 2 diabetic patients with no known CAD was selected. Patients with coronary stents or CABG surgery were excluded. Information regarding risk factors for CAD was collected for all patients. All patients in the study provided written consent. Mean age of the cohort was $60.3 \pm 8.2$, with $78.85 \%$ being male. All patients underwent a CT coronary angiogram (CTCA). CTCA was reconstructed at the best diastolic phase, and plaques were categorised as non-significant (causing $<50 \%$ stenosis) or significant (causing $>50 \%$ or $70 \%$ stenosis). Categorical variables were presented as percentages and continuous variables as mean \pm standard deviation.

\section{Results}

We found only $16.9 \%$ of the cohort had normal coronary arteries, $80.2 \%$ had at least one plaque causing nonsignificant stenosis (1-49\%). 56.3\% of the cohort had at least one plaque causing stenosis of $>50 \%$. $33.8 \%$ patients had at least one plaque causing stenosis of
$>70 \%$. We also found that this cohort had per patient, $1.17 \pm 1.9$ non-calcified plaques (NCP), $3.06 \pm 3.33$ calcified plaques, and 1.06 \pm 1.4 mixed plaques (MP).

\section{Conclusions}

Our study demonstrates that more than half of asymptomatic diabetic patients have at least one plaque causing stenosis of $>50 \%$ and more than a third have at least one plaque causing stenosis of $>70 \%$. This suggests a revision of guidelines is needed for CAD screening in type 2 diabetic patients in order to reduce mortality and morbidity. A limitation of our study is that we have not taken into account duration of diabetes and control of blood sugar levels during this time.

\section{Author details}

${ }^{1}$ University College London Medical School, UK. ${ }^{2}$ Clinical Imaging and Research Centre Wellington Hospital, London, UK. ${ }^{3}$ Imperial College, London, UK. ${ }^{4}$ Middlesex University, London, UK.

Published: 9 July 2012

doi:10.1186/1753-6561-6-S4-O20

Cite this article as: Patel et al:: An evaluation of coronary artery plaque burden in asymptomatic type 2 diabetics using dual-source CT coronary angiography. BMC Proceedings 2012 6(Suppl 4):O20.

${ }^{1}$ University College London Medical School, UK

Full list of author information is available at the end of the article

(C) 2012 Patel et al; licensee BioMed Central Ltd. This is an Open Access article distributed under the terms of the Creative Commons 